\title{
Aprendizados Sobre o Impacto do COVID-19 nas Organizações
}

\author{
Teaching Covid-19's Impact on Businesses
}

\author{
Leonardo Marques' ${ }^{\circ}$ \\ Paula C. P. de Souza Chimenti ${ }^{1,2}$ - \\ Wesley Mendes-da-Silva ${ }^{3,4} \bullet$
}

\section{MARCADO NA HISTÓRIA}

2020 ficará marcado na História como um ano de crise. Um ano difícil, de muita perda, muita incapacidade e muita tristeza. Nosso special issue sobre o COVID-19 é uma tentativa de extrair algo deste tempo devastador. De refletir sobre os desafios que vivemos e o que aprendemos com eles.

Numa palestra proferida em 2020, Jared Diamond, autor de Guns, germs and steel (Diamond, 1999), declarou que o coronavírus não era o maior desafio da humanidade. Os grandes desafios do planeta para ele são a dificuldade de lidar com a diversidade e a inclusão, o uso insustentável dos recursos do planeta e a crescente desigualdade que marginaliza grande parte da população mundial. $\mathrm{Na}$ visão do autor, o COVID-19 poderia talvez nos ajudar a lidar com esses problemas, dependendo de como reagíssemos. Isto porque a crise gerada por esta pandemia deixou claro para todos que fronteiras são cada vez mais fluidas, que habitamos um único ecossistema e que tudo o que fazemos tem impacto em nós mesmos, mas também nos outros.

Partilhamos dessa visão. E percebemos que, diante de uma necessidade de transformação táo profunda, o papel da educação é absolutamente fundamental. Uma educação renovada, que emerge da crise sem querer dar todas as respostas certas, pois já sabemos que elas não existem, mas

1. Universidade Federal do Rio de Janeiro, Instituto COPPEAD de Administração, Rio de Janeiro, RJ, Brasil. 2. Editora Associada da RAC - Revista de Administração Contemporânea.

3. Fundação Getulio Vargas, Escola de Administração de Empresas de São Paulo, São Paulo, SP, Brasil. 4. Editor-chefe da RAC - Revista de Administração Contemporânea. nos ajudando a fazer as perguntas e a refletir sobre o que de fato importa.

A chamada de casos para ensino nasceu de uma inquietação que compartilhávamos sobre o que o futuro nos reservava e que aprendizados poderíamos extrair de tudo que estava acontecendo. Recebemos 45 trabalhos de qualidade. Seis meses depois, os trabalhos que constituem esta edição nos mostram muitos dilemas relevantes, muitas incertezas e muitos novos conhecimentos. De fato, aprendemos muito.

Aprendemos sobre os desafios da aceleração abrupta do home office com a Chianca Softwares. Aprendemos sobre o valor e a coragem do empreendedor com Sorela, Casa Porto e Wanderlust. Aprendemos sobre reconfiguraçáo de negócios e canais de distribuição com IGT, Pescaria Argo e Legrias. Aprendemos que nos organizamos cada vez mais em plataformas que conectam grupos distintos com Moeda Seeds, Diogo Nogueira e DocPass. Aprendemos que essas plataformas fazem parte de ecossistemas cada vez mais complexos e interligados com HCFMUSP e Coppead. E aprendemos sobre a responsabilidade ética de gerir negócios nesses ambientes com Magalu e Universidade Federal da Paraíba. 


\section{AÇÃO E REAÇÃO}

Cada caso que compóe esta special issue oferece uma rica reflexão sobre como negócios nas mais diversas áreas sofreram o black swan (Taleb, 2007) chamado pandemia COVID-19, e como tiveram que reagir para se adaptar e atender ao imperativo da sobrevivência, mesmo em tempos tão difíceis.

Boa parte dos casos foca nas pequenas empresas e como foram particularmente afetadas pela pandemia. $\mathrm{O}$ caso de abertura trata de uma transformaçáo que afetou em algum grau a grande maioria das empresas: a guinada ao home office. O caso da Chianca Softwares (Alves, Amorim, \& Bezerra, 2021) discute o ciclo completo, começando pelos desafios de implementar trabalho remoto em pleno momento de descoberta do que era a pandemia e seus reais riscos, passando pela adaptação ao home office, e finalmente discutindo até que ponto este momento de exceçáo abriu uma oportunidade para empresas repensarem seu formato de trabalho: vale a pena voltar ao trabalho presencial ou seria melhor pensar em um modelo híbrido? Na sequência, o caso da maior expoente do mercado de açóes brasileiro - a Magazine Luiza (Passos, Meneghini, Gama, \& Lana, 2021) - mostra como empatia, ética e gestáo dos recursos humanos se tornaram um imperativo durante as aceleradas curvas de contaminaçóes, internações e óbitos provocadas pelo COVID-19. O caso mostra que a pandemia não só provocou reformataçóes em negócios de pequeno porte, mas exigiu adaptação de grandes corporaçôes.

A seguir, temos quatro casos que dão destaque ao papel do empreendedor à frente de seus negócios. $\mathrm{O}$ caso Wanderlust (Pinho, Monteiro, \& Binhote, 2021) mostra os desafios de uma empreendedora digital focada em turismo em um momento de restrição quase total de circulação. Como revisar sua proposta de marketing para se manter relevante durante este período? Já no caso Sorela (Erthal \& Sacramento, 2021), um jovem empreendedor no negócio de venda de veículos vê na pandemia a oportunidade de avançar na digitalizaçáo que antes era refratada por seus sócios mais velhos. Ou quem sabe aproveitar o momento de reflexão para, a partir da lógica de job crafting, mergulhar em novo projeto born digital? No caso da Casa Porto (Vinhas \& Lopes, 2021), o empreendedor precisa apertar os cintos do negócio, enquanto luta para seguir pagando sua equipe. E, finalmente, o caso Facile/Ligferv (Silva, Guimarães, \& Castro, 2021) oferece ambiente empírico para aplicação das consagradas teorias de empreendedorismo causation e effectuation para explicar os desafios de um empreendedor de Minas Gerais que precisa reinventar o modelo de negócios de sua inovadora cafeteira para seguir adiante.
Os três próximos casos focam na reorganização da cadeia de valor desde a gestão de fornecedores até a escolha de canais de distribuição. O Caso IGT (Fonseca \& Rocha, 2021) trata de um fabricante e distribuidor de kit de conversão para gás natural. Quantas empresas tiveram que rever seus planos de expansão, internacionalização e diversificação de uma hora para a outra a partir da pandemia? O caso explora aspectos de empreendedorismo para decidir como reorganizar seu supply chain e alinhá-lo com a comunicação ao cliente - agravada pela politizaçáo do papel da China na pandemia. De forma similar, a Pescaria Argo (Cavalcanti, Avelar, Amaral, \& Freitas, 2021) sofre com a ruptura na demanda quando as fronteiras internacionais se fecham. $O$ caso trabalha ferramentas financeiras para apoio na tomada de decisão e gestão de custos. A identificaçáo dos custos relevantes se torna crucial para decidir entre alternativas de canais de distribuição para escoar seus produtos. Fechando este conjunto de casos que tratam da reconfiguraçáo de negócios, no caso Legrias (Silva, Limongi, Gomes, \& Pimenta, 2021) vemos novamente uma pequena empresa de produção de chocolate surpreendida pela pandemia, revisando o path to market e os custos de canais alternativos em meio a tantas incertezas.

$\mathrm{Na}$ sequência desta edição, expandimos nosso olhar para plataformas e ecossistemas. No caso sobre o músico Diogo Nogueira (Rodrigues, Kamlot, \& Carvalho, 2021), o modelo de plataforma é usado para discutir inovação e reinvenção em um dos setores mais afetados pelo COVID-19: música e entretenimento. A reflexão sobre os desafios desse músico de renome no cenário nacional permite a compreensáo das propriedades das plataformas digitais, como multilateralidade, efeitos de rede e potencial de aprisionamento dos usuários. No caso DocPass (Moreira, Freitas, Brandão, \& Araújo, 2021), podemos aprender com o audacioso movimento da empresa de telemedicina Conexa, que já operava na modalidade B2B antes da pandemia, e que decide explorar a mudança da legislação para preencher a lacuna do atendimento de telemedicina B2C com o lançamento da DocPass. O caso permite a discussão dos efeitos de rede em um setor altamente relevante e em franca expansão. Já no caso Moeda Seeds (Kanegae, Cernev, \& Diniz, 2021), o framework de ecossistema é usado para discutir os desafios de uma plataforma digital de inovação social baseada em blockchain. $\mathrm{O}$ caso oferece uma reflexão sobre o papel que o blockchain pode desempenhar em negócios de impacto, assim como os desafios de uma empreendedora social tentando revisar o posicionamento do seu marketplace com sensibilidade para dialogar com parceiros em condiçóes de vulnerabilidade, ainda mais durante uma pandemia.

Por fim, aprendemos que ecossistemas se tornam cada vez mais complexos e interligados com dois casos voltados para a saúde e dois casos focados em educação 
- provavelmente os dois setores mais desafiados em uma crise como a pandemia do COVID-19. O caso do Hospital das Clínicas da Faculdade de Medicina da USP (Pedroso, Pires, Malik, \& Pereira, 2021) explora a adaptação da governança como resposta a uma crise, começando na readequação da capacidade produtiva de uma operação tradicionalmente considerada pouco resiliente e chegando à reflexão de como uma pandemia sem precedentes pode ajudar na gestáo de crises futuras. O caso seguinte trata do Sistema Indústria (Andrade, Sousa-Filho, Almeida, \& Câmara, 2021) adaptando-se para atender a demanda de curto prazo do setor desaúde. Acomplexa governançadeseteinstituições diferentes se faz necessária para enfrentar a crise. Já o interessante caso da Universidade Federal da Paraíba (Teixeira-de-Carvalho, Dias, \& Bispo, 2021) analisa a virtualizaçáo do ensino, enfatizando os desafios em larga escala de uma universidade federal: a gestáo do calendário com incertezas, corpo docente e discente fragilizados, e as diferenças entre graduação e pósgraduaçáo. E, por fim, o caso convidado sobre o Coppead UFRJ (Campos, Tavares, Chimenti, \& Marques, 2021), que complementa a discussão sobre os desafios da virtualização do ensino, com uma análise de cenários que permite

\section{REFERÊNCIAS}

Alves, T. L. de L., Amorim, A. F. A., \& Bezerra, M. C. C. (2021). "Not one less"! Adaptation to the home office in times of COVID-19. Revista de Administração Contemporânea, 25(spe), e200234. https://doi.org/10.1590/1982-7849rac2021200234.en

Andrade, R. J. C., Sousa-Filho, J. M., Almeida, F. E. B., \& Câmara, S. F. (2021). Pandemic fights in a network! COVID-19 challenges in northeast Brazil. Revista de Administração Contemporânea, 25(spe), e200256. https://doi.org/10.1590/1982-7849rac2021200256.en

Campos, R. D., Tavares, E., Chimenti, P. C. P. de S., \& Marques, L. (2021). Challenges for the future of education brought by the pandemic: The Coppead case. Revista de Administraçấo Contemporânea, 25(spe), e210062. https://doi.org/10.1590/1982-7849rac2021210062.en

Cavalcanti, J. M. M., Avelar, E. A., Amaral, F. R. C. B. do, \& Freitas, K. A. (2021). The sea is getting' rough: Analysis of incremental costs during the pandemic. Revista de Administração Contemporânea, 25(spe), e200227. https://doi.org/10.1590/1982-7849rac2021200227.en

Diamond, J. (1999). Guns, germs, and steel. The fates of human societies. New York: W. W. Norton \& Company revisitar uma crise de proporções nunca vistas como fonte de aprendizado e reflexão para a revisão do método de ensino no médio e longo prazo.

\section{DE VOLTA PARA A SALA DE AULA}

A educação é a chave para o futuro. Pode nos ajudar a ressignificar o trabalho, o mérito, o poder. Pode lançar luz e ideias sobre debates que, de outra forma, ficariam superficiais, repletos de certezas não fundamentadas ou, pior, de mentiras que se passam por verdades. Nosso trabalho como educadores é tremendo. E precisamos crescer com a ocasião.

Neste início de 2021, sabemos que ainda temos muito que aprender. Mas temos certeza de que o conjunto de casos de ensino apresentado aqui propiciará o debate franco e aberto que uma sala de aula precisa ter para cumprir sua missão de questionar, provocar e educar.

Bons debates! Excelente aprendizado! E um 2021 muito melhor!

Erthal, A., \& Sacramento, I. (2021). Rafael and the Sorela Vehicles case: The pandemic as an enhancer of job crafting. Revista de Administraçâo Contemporânea, 25(spe), e200252. https://doi.org/10.1590/1982-7849rac2021200252.en

Fonseca, L., \& Rocha, A. (2021). Rethinking offshoring and international marketing strategies during the COVID-19 pandemic. Revista de Administração Contemporânea, 25(spe), e200216. https://doi.org/10.1590/1982-7849rac2021200216.en

Kanegae, L. C., Cernev, A. K., \& Diniz, E. (2021). Moeda Seeds: E-commerce and crypto solutions for development. Revista de Administração Contemporânea, 25(spe), e200224. https://doi.org/10.1590/1982-7849rac2021200224.en

Moreira, C. S. da C., Freitas, F. D., Brandão, C., \& Araujo, C. A. S. (2021). From Conexa to Docpass: The competitive environment of telemedicine platforms. Revista de Administração Contemporânea, 25(spe), e200238. https://doi.org/10.1590/1982-7849rac2021200238.en

Passos, A. P. P., Meneghini, E. M. P., Gama, M. A. B., \& Lana, J. (2021). "Magalu has it": Social, political and market strategies during COVID-19. Revista de Administração Contemporânea, 25(spe), e200233. https://doi.org/10.1590/1982-7849rac2021200233.en 
Pedroso, M. C., Pires, J. T., Malik, A. M., \& Pereira, A. J. R. (2021). HCFMUSP: Resilience in response to the COVID-19 pandemic. Revista de Administração Contemporânea, 25(spe), e200245. https://doi.org/10.1590/1982-7849rac2021200245.en

Pinho,V.M.de,Monteiro,J., \&Binhote,J.(2021). Wanderlustwithout wandering: Managing a travel blog during the Covid-19. Revista de Administraçáo Contemporânea, 25(spe), e200243. https://doi.org/10.1590/1982-7849rac2021200243.en

Rodrigues, M. A. de S., Kamlot, D., \& Carvalho, A. V. de (2021). Pandemic, samba, and Diogo Nogueira's livestreams: Platform management challenges. Revista de Administração Contemporânea, 25(spe), e200225. https://doi.org/10.1590/1982-7849rac2021200225.en

Silva, J. P. M., Guimarães, L. O., \& Castro, J. M. (2021). Facile/LigFerv: Hot water in three seconds, thirty years of entrepreneurial process. Revista de Administração Contemporânea, 25(spe), e200221. https://doi.org/10.1590/1982-7849rac2021200221.en

\section{Autoria}

\section{Leonardo Marques}

Universidade Federal do Rio de Janeiro, Instituto COPPEAD de Administração

Rua Pascoal Lemme, no 355, Cidade Universitária, 21941-918, Rio de Janeiro, RJ, Brasil.

E-mail: leo.marques@coppead.ufrj.br

(D) https://orcid.org/0000-0001-8960-8191

\section{Paula Castro Pires de Souza Chimenti*}

Universidade Federal do Rio de Janeiro, Instituto COPPEAD de Administração

Rua Pascoal Lemme, no 355, Cidade Universitária, 21941-918, Rio de Janeiro, RJ, Brasil.

E-mail: paula.chimenti@coppead.ufrj.br

(D) https://orcid.org/0000-0002-6492-4072

\section{Wesley Mendes-da-Silva}

Fundação Getulio Vargas, Escola de Administração de Empresas de Sáo Paulo.

Rua Itapeva, 474, $8^{\circ}$ andar, 01332-000, São Paulo, SP, Brasil.

E-mail: rac.wesley.mendes@gmail.com

(i) https://orcid.org/0000-0002-5500-4872

* Autora Correspondente

\section{Conflito de Interesses}

Os autores informaram que não há conflito de interesses.
Silva, A. L. B., Limongi, R., Gomes, A. C., \& Pimenta, D. (2021). Chocolates and 'Legrias': Dilemma of the best sales channel. Revista de Administração Contemporânea, 25(spe), e200235. https://doi.org/10.1590/1982-7849rac2021200235.en

Taleb, N. N. (2007). The black swan. The impact of the highly improbable. New York: Random House.

Teixeira-de-Carvalho, D. L., Dias, J. J. L., Junior, \& Bispo, A. C. K. de A. (2021). Our calendar stopped! The organizational change at UFPB due to COVID-19. Revista de Administração Contemporânea, 25(spe), e200249. https://doi.org/10.1590/1982-7849rac2021200249.en

Vinhas, V. Q., \& Lopes, A. L. S. V. (2021). Stay at home, Casa Porto delivers: Humanized entrepreneurship during pandemic. Revista de Administração Contemporânea, 25(spe), e200253. https://doi.org/10.1590/1982-7849rac2021200253.en

\section{Direitos Autorais}

A RAC detém os direitos autorais deste conteúdo.

\section{Verificação de Plágio}

A RAC mantém a prática de submeter todos os documentos aprovados para publicação à verificação de plágio, mediante o emprego de ferramentas específicas, e.g.: iThenticate.

\section{CORPO EDITORIAL CIENTÍFICO E EQUIPE EDITORIAL PARA ESTA EDIÇÃO:}

\section{Conselho Editorial}

Anielson Barbosa da Silva (UFPB, João Pessoa, Brasil) Antonio Carlos Gastaud Maçada (UFRGS, Porto Alegre, Brasil) Ely Laureano Paiva (FGV, São Paulo, Brasil)

Rogério Hermida Quintella (NPGA/UFBA, Salvador, Brasil)

Valmir Emil Hoffmann (UnB, Brasília, Brasil)

Wesley Mendes-da-Silva (FGV/ EAESP, São Paulo, Brasil)

\section{Editor-chefe}

Wesley Mendes-da-Silva (FGV/ EAESP, São Paulo, Brasil)

\section{Editores Associados}

Eduardo da Silva Flores (FEA/USP, São Paulo, Brasil)

Emílio José M. Arruda Filho (UNAMA, Belém, Brasil)

Fabio Caldieraro (EBAPE/FGV, São Paulo, Brasil)

Gilnei Luiz de Moura (UFSM, Santa Maria, Brasil)

Henrique Castro Martins (IAG PUC-Rio, Rio de Janeiro, Brasil) 
Ismael Ali Ali (Kent State University, Ohio, EUA)

Marcus Cunha Junior (University of Georgia, EUA)

Paula Castro Pires de Souza Chimenti (UFRJ/Coppead, Rio de Janeiro, Brasil)

Paulo César Matui (UniGranRio, Rio de Janeiro, Brasil)

\section{Corpo Editorial Científico}

André Luiz Maranhão de Souza-Leão (UFPE, Recife, Brasil)

Aureliano Angel Bressan (CEPEAD/UFMG, Belo Horizonte, Brasil)

Bryan Husted (York University, Canadá)

Carlos M. Rodriguez (Delaware State University, EUA)

Cristiana Cerqueira Leal (Universidade do Minho, Portugal)

Diógenes de Souza Bido (Mackenzie, São Paulo, Brasil)

Erica Piros Kovacs (Kelley School of Business/Indiana University, EUA)

Elin Merethe Oftedal (University of Stavanger, Noruega)

Fábio Frezatti (FEA/USP, São Paulo, Brasil)

Felipe Monteiro (INSEAD Business School, EUA)

Howard J. Rush (University of Brighton, Reino Unido)

James Robert Moon Junior (Georgia Institute of Technology, EUA)

John L. Campbell (University of Georgia, EUA)

José Antônio Puppim de Oliveira (United Nations University,

Yokohama, Japáo)

Julián Cárdenas (Freie Universität, Berlin, Alemanha)
Lucas A. B. de Campos Barros (FEA/USP, São Paulo, Brasil)

Luciano Rossoni (UniGranRio, Rio de Janeiro, Brasil)

M. Philippe Protin (Université Grenoble Alpes, França)

Paulo Estevão Cruvinel (Embrapa Instrumentação, São Carlos, Brasil)

Rodrigo Bandeira de Mello (Merrimack College, EUA)

Rodrigo Verdi (MIT Massachusetts Institute of Technology, Cambridge, EUA)

Valter Afonso Vieira, (UEM, Maringá, Brasil)

Wagner Kamakura (Jones Graduate School of Business, Rice University, Houston, EUA)

\section{Editoração}

Diagramação e normas da APA: Kler Godoy (ANPAD, Maringá, Brasil); Simone L. L. Rafael (ANPAD, Maringá, Brasil).

Periodicidade: Publicação contínua.

Circulação: Acesso totalmente gratuito.

\section{Indexadores e Diretórios}

Scielo, Redalyc, DOAJ, Latindex, Cengage/GALE, Econpapers, EBSCO, MIAR, Proquest, SPELL, Cabell's, Ulrichs, CLASE, DIADORIM, ERIHPlus, EZB, EuroPub, OasisBR, WorldWideScience, Google Scholar, Capes/Qualis, Citefactor.org, Index Copernicus International, Sherpa Romeo. 